\title{
Role of Hidden Slow Degrees of Freedom in the Fluctuation Theorem
}

\author{
J. Mehl,${ }^{1}$ B. Lander, ${ }^{2}$ C. Bechinger,${ }^{1,3}$ V. Blickle, ${ }^{1,3}$ and U. Seifert ${ }^{2}$ \\ ${ }^{1}$ 2. Physikalisches Institut, Universität Stuttgart, Pfaffenwaldring 57, 70569 Stuttgart, Germany \\ ${ }^{2}$ II. Institut für Theoretische Physik, Universität Stuttgart, Pfaffenwaldring 57, 70550 Stuttgart, Germany \\ ${ }^{3}$ Max-Planck-Institute for Intelligent Systems, Heisenbergstrasse 3, 70569 Stuttgart, Germany
}

\begin{abstract}
The validity of the fluctuation theorem for entropy production as deduced from the observation of trajectories implicitly requires that all slow degrees of freedom are accessible. We experimentally investigate the role of hidden slow degrees of freedom in a system of two magnetically coupled driven colloidal particles. The apparent entropy production based on the observation of just one particle obeys a fluctuation theorem-like symmetry with a slope of 1 in the short time limit. For longer times, we find a constant slope, but different from 1 . We present theoretical arguments for a generic linear behavior both for small and large apparent entropy production but not necessarily throughout. By fine-tuning experimental parameters, such an intermediate nonlinear behavior can indeed be recovered in our system as well.
\end{abstract}

PACS numbers: 05.70.Ln, 05.40.--a, 82.70.Dd

Introduction.-Basic concepts of thermodynamics and statistical physics implicitly rest on a separation of all degrees of freedom into observable and non-observable ones. Heat exchange, for example, is associated with the myriads of fast degrees of freedom which are not resolved dynamically, whereas work typically involves a few controlled, slow degrees of freedom. In systems without a clear-cut time-scale separation, ambiguities and inconsistencies may arise if such concepts are still applied naively. Here we explore this issue for one of the arguably most relevant concepts, entropy production $\Delta s_{\text {tot }}$, for nonequilibrium steady states (NESS). For such states, the fluctuation theorem (FT) refers to a remarkable symmetry that quantifies the probability $p$ of observing trajectories with negative total entropy production as

$$
\ln \left[p\left(\Delta s_{\text {tot }}\right) / p\left(-\Delta s_{\text {tot }}\right)\right]=\alpha \Delta s_{\text {tot }}
$$

with $\alpha=1$ and Boltzmann's constant set to unity [1 5 .

The FT has been proven for two types of dynamics. First, for deterministic dynamics the proof rests on the chaotic hypothesis, including time reversibility and a phase-space contraction associated with dissipation [2]. Second, in stochastic dynamics the FT requires the concept of entropy production along trajectories and can be proven for Markovian systems $[3]$ [ $]$. The latter dynamics applies to experiments on driven colloidal particles [6, 7] and a harmonic oscillator coupled to a thermal bath [8]. Experimental tests of FT-like symmetries have also been reported for Rayleigh-Bénard convection [9], turbulent flow 10], granular matter [11], and self-propelled particles [12]. For these systems the appropriate class of dynamics is less obvious and hence the status regarding the assumptions of the FT is unclear a priori. One should also appreciate that the measured observable for some of these systems is typically not $\Delta s_{\text {tot }}$ directly, but rather some dimensionful quantity, like, e.g., the injected or dissipated work [11, 13], which requires a temperature for a unique conversion to entropy. Strictly speaking, the
FT is thus verified only if this temperature can be determined independently and if it leads to $\alpha=1$. Complementary, in more recent reports, the validity of the FT (with $\alpha=1$ ) is assumed and used to gain information about such a dimensionful factor connecting the actual observable with entropy production 14, 15].

For stochastic dynamics, the proof of the FT with $\alpha=1$ rests on a time-scale separation. Fast degrees of freedom contribute to an effectively white noise leading then to a Markovian dynamics of the slow degrees of freedom. Entropy production can be deduced from observing the dynamics of all slow degrees of freedom. If some of these degrees of freedom are not, or cannot, be observed the inferred entropy production is only an apparent one for which the status of an FT-like symmetry is unclear $a$ priori. Theoretical efforts to describe coarse-graining in general have been restricted so far to the case of well separated time scales [16 18], and how such coarse-graining affects bounds on dissipated work 19]. In the framework of electronic devices FT-like symmetries for currents have been discussed in Refs. 20 22].

In the present paper, we investigate the role of hidden slow degrees of freedom on apparent entropy production for a paradigmatic system with two magnetically coupled driven colloidal particles.

Apparent Entropy Production.- The total entropy production $\Delta s_{\text {tot }}$ is given by the sum of the entropy changes of the heat bath and system [5]

$$
\Delta s_{\text {tot }}=Q / T+\ln \left[p^{\mathrm{s}}\left(\mathbf{x}^{0}\right) / p^{\mathrm{s}}\left(\mathbf{x}^{t}\right)\right]
$$

where $Q=\int_{0}^{t} d \tau \sum_{i=1}^{n} \dot{x}_{i}(\tau) F_{i}(\mathbf{x}(\tau))$ is the heat transfer of all $n$ degrees of freedom $\mathbf{x} \equiv\left(x_{1} \ldots x_{n}\right)$ to the solvent at temperature $T$. Here, $\dot{x}_{i}$ is the actual velocity and $F_{i}$ is the total force acting on the $i^{\text {th }}$ degree of freedom. The change of the system's entropy includes the stationary probability distribution $p^{\mathrm{s}}\left(\mathbf{x}^{0}\right)\left(p^{\mathrm{s}}\left(\mathbf{x}^{t}\right)\right)$ of finding the initial (final) state of the system along the trajectory of length $t$. In a NESS, the system satisfies the station- 
ary Smoluchowski equation $0=-\sum_{i} \partial_{x_{i}} j_{i}^{\mathrm{s}}(\mathbf{x})$, where the probability current $j_{i}^{\mathrm{s}}(\mathbf{x})=p^{\mathrm{s}}(\mathbf{x}) \nu_{i}^{\mathrm{s}}(\mathbf{x})$ is given as the product of $p^{\mathrm{s}}$ and the mean local velocity [5]

$$
\nu_{i}^{\mathrm{s}}(\mathbf{x}) \equiv D_{0}\left[F_{i}(\mathbf{x}) / T-\partial_{x_{i}} \ln p^{\mathrm{s}}(\mathbf{x})\right]
$$

with $D_{0}$ the bare diffusivity. Multiplying Eq. (3) with $\dot{x}_{i}$ and integrating over time yields the total entropy production as given in Eq. (2),

$$
\Delta s_{\text {tot }}=\int_{0}^{t} d \tau \sum_{i=1}^{n} \dot{x}_{i}(\tau) \nu_{i}^{\mathrm{s}}(\mathbf{x}(\tau)) / D_{0},
$$

where the sum involves all $n$ degrees of freedom $\mathbf{x}$. If only the first $\tilde{n}$ of these, $\tilde{\mathbf{x}} \equiv\left(x_{1} \ldots x_{\tilde{n}}\right)$, are accessible, an observer is forced to deduce all information from these trajectories. The actual velocities $\dot{\tilde{\mathbf{x}}}$ can still be measured correctly, whereas the mean local velocity obtained from the accessible trajectories is

$$
\tilde{\nu}_{i}^{\mathrm{s}}(\tilde{\mathbf{x}}) \equiv \int \nu_{i}^{\mathrm{S}}(\mathbf{x}) p^{\mathrm{s}}(\hat{\mathbf{x}} \mid \tilde{\mathbf{x}}) d \hat{\mathbf{x}}
$$

with the conditional probability $p^{\mathrm{s}}(\hat{\mathbf{x}} \mid \tilde{\mathbf{x}})$ for $\hat{\mathbf{x}} \equiv$ $\left(x_{\tilde{n}+1}, \ldots, x_{n}\right)$ at fixed $\tilde{\mathbf{x}}$. Hence, the apparent entropy production becomes

$$
\Delta \tilde{s}_{\text {tot }}=\int_{0}^{t} d \tau \sum_{i=1}^{\tilde{n}} \dot{x}_{i}(\tau) \tilde{\nu}_{i}^{\mathrm{S}}(\tilde{\mathbf{x}}(\tau)) / D_{0},
$$

where the sum runs over the $\tilde{n}$ accessible degrees of freedom only. In this Letter, we investigate the conditions under which this quantity obeys a FT-like symmetry.

Experiment.-We have created two non-overlapping toroidal traps with radius $R=3.5 \mu \mathrm{m}$ and a centercenter distance of $17 \mu \mathrm{m}$ by a single laser beam $(\lambda=$ $1070 \mathrm{~nm}$ ) which was deflected on a galvanometric mirror unit (for details refer to [23, 24]). Each trap contained a single paramagnetic colloidal particle with a $2.6 \mu \mathrm{m}$ radius (Microparticles, Berlin) and labeled by an index $i=1,2$ (see Fig. 1(a)). The traps are approximately $50 \mu \mathrm{m}$ away from the lower surface; therefore hydrodynamic interactions with the walls are negligible 25]. The scanning frequency was adjusted to $41 \mathrm{~Hz}$, which leads to quasistatic tangential forces $f_{i}$ acting on the $i^{\text {th }}$ particle along the toroidal traps whose amplitude depends on the laser intensity. In our experiments, the time for a full revolution of each particle was adjusted to $10 \mathrm{~s}$. Synchronized with the scanning motion of the laser beam, its intensity was sinusoidally modulated with an acoustooptic modulator which finally leads to an effective optical potential $U_{i}\left(x_{i}\right)=V_{i}\left(x_{i}\right)-f_{i} x_{i}$ with $V_{i}\left(x_{i}\right)=V_{i}^{0} \sin \left(x_{i}\right)$, where $x_{i}$ is the particle position along the trap circumference in units of $\pi R$ (see Fig. 1(a)). Accordingly, both particles reach NESS, where $f_{i}$ and $V_{i}^{0}$ can be controlled independently.

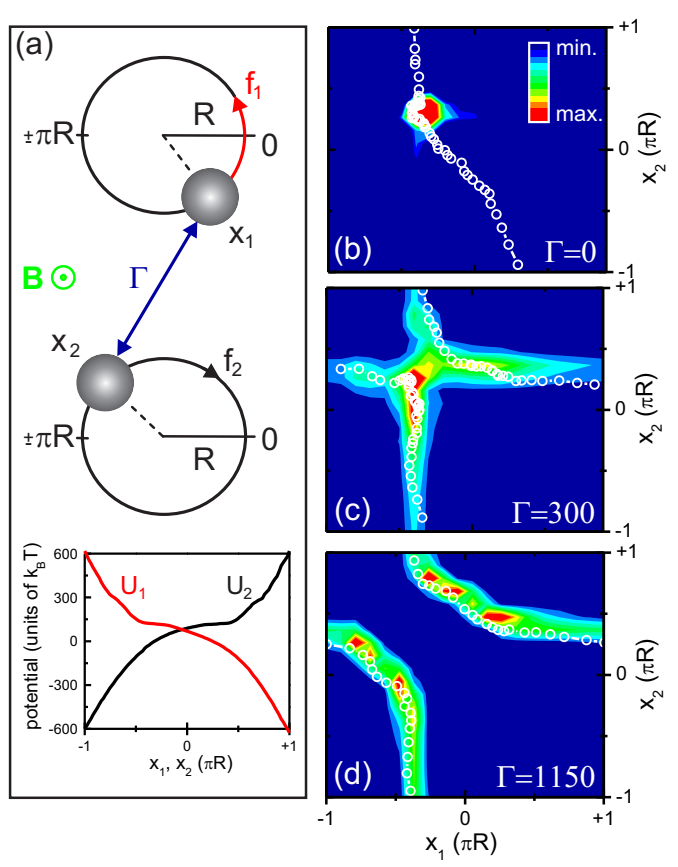

FIG. 1: (color online) (a) Schematic representation of the system and measured tilted potentials $\left(V_{1}^{0}=V_{2}^{0}=181 \mathrm{k}_{\mathrm{B}} \mathrm{T}\right.$ and $f_{1}=-f_{2}=57 \mathrm{kB}$ T/ $\mu \mathrm{m}$ calculated via 26]). (b)-(d) Stationary probability distribution $p^{\mathrm{s}}\left(x_{1}, x_{2}\right)$ for different plasma parameters $\Gamma$. The white circles indicate typical trajectories.

A coupling between the two NESS is obtained by a static homogeneous magnetic field $B$ applied perpendicular to the sample plane. This field induces magnetic moments $m$ to the particles leading to a repulsive dipolar particle interaction $W\left(x_{1}, x_{2}\right)=\left(\mu_{0} / 4 \pi\right) m^{2} / r^{3}\left(x_{1}, x_{2}\right)$. Here, $\mu_{0}$ is the magnetic constant and $r$ the particle distance. For small magnetic fields $(B \leqslant 40 \mathrm{mT})$, as in our experiments, the magnetic moment scales as $m \approx\left(\gamma m_{0} / 3\right) B$ with $\gamma=30 \mathrm{~T}^{-1}$ and $m_{0}=5.9 \times$ $10^{-13} \mathrm{Am}^{2}$ 28]. The strength of the dipolar coupling can be conveniently characterized by a dimensionless plasma parameter $\Gamma \equiv \Delta W /\left(k_{\mathrm{B}} T\right)$, where $\Delta W$ corresponds to the difference of the coupling at the smallest and largest particle distance $r$. Additional particle interactions, e.g., hydrodynamic coupling or optical binding, are negligible at the chosen trap separations as confirmed by the independent motion of the particles in the absence of a magnetic field.

Results.-We investigate the effect of coupling by preparing two identical NESS, where the potential minima face each other. Figures (b)-(d) show the stationary probability distribution $p^{\mathrm{s}}\left(x_{1}, x_{2}\right)$ as color coded background and as white circles one exemplary trajectory. In the absence of coupling, the peak in $p^{\mathrm{s}}$ corresponds to the flattest part in the potentials, where the parti- 

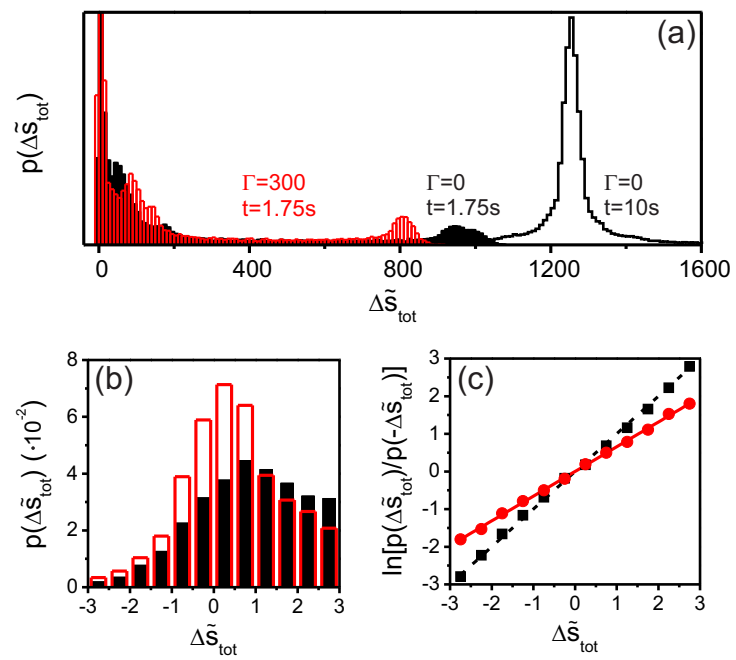

FIG. 2: (color online) (a) Distribution of the apparent entropy production $p\left(\Delta \tilde{s}_{\text {tot }}\right)$ for different trajectory lengths $t$ and plasma parameters $\Gamma$. (b) Section of previous histograms around $\Delta \tilde{s}_{\text {tot }}=0$. (c) Corresponding $\ln \left[p\left(\Delta \tilde{s}_{\text {tot }}\right) / p\left(-\Delta \tilde{s}_{\text {tot }}\right)\right]$ as a function of $\Delta \tilde{s}_{\text {tot }}$. The dashed black line has the theoretically predicted slope 1 , whereas the red line is a linear fit with slope $\alpha=0.65$.

cles slow down and therefore are most likely to be found (see Fig. 1(b)). Under strong coupling conditions (see Fig. 1(d)), the repulsion hinders the particles from coming close to each other. The peak of $p^{\mathrm{s}}$ vanishes since the approach of one particle kicks the other one away, leading to motion like a Newton's cradle. In the intermediate regime (see Fig. 1(c)), the coupling interaction is comparable with the energy loss acquired while the particle moves along half a circle. Here the full interplay between drift, diffusion, and interaction has to be taken into account and we expect the influence of hidden degrees of freedom on the FT to be most prominent. Therefore, we concentrate on $\Delta \tilde{s}_{\text {tot }}$ associated with the motion of only the first particle, which represents the observed degree of freedom, whereas the coupling allows us to uniquely control the influence of the second particle, which acts as a hidden degree of freedom.

The black histograms (closed bars and line) in Fig. 2(a) show the distribution of the apparent entropy production $p\left(\Delta \tilde{s}_{\text {tot }}\right)$ in the absence of coupling obtained for trajectories of length $t=1.75 \mathrm{~s}$ and $10 \mathrm{~s}$, respectively. The peaked distribution shifts with elapsing time to the right with peak height maxima occurring at positions which correspond to the energy loss associated with full revolutions of the particle, $2 \pi R f=1250 \mathrm{k}_{\mathrm{B}} \mathrm{T}$. To investigate the FT, rare events with negative entropy production have to be sampled with high accuracy. This constrains
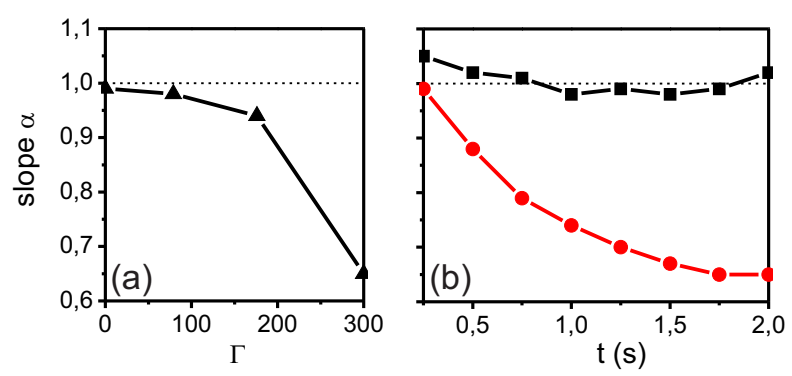

FIG. 3: (color online) (a) Slope $\alpha$ vs plasma parameter $\Gamma$ for $t=1.75 \mathrm{~s}$. (b) Slope $\alpha$ for different trajectory lengths $t$. The black squares correspond to $\Gamma=0$ and the red dots to $\Gamma=300$. The deviation of the black squares from $\alpha=1$ (black dashed line) determines the statistical errors to be less than $5 \%$.

the maximal trajectory length $t$ to approximately $2 \mathrm{~s}$ and the range within the FT can be tested to \pm 3 . Figure2(b) shows this section of the black (closed bars) histogram around $\Delta \tilde{s}_{\text {tot }}=0$. The excellent agreement between the logarithm of the probability ratio $p\left(\Delta \tilde{s}_{\text {tot }}\right) / p\left(-\Delta \tilde{s}_{\text {tot }}\right)$, black squares in Fig. 2(c), and the black dashed line with a slope of 1 confirms the validity of Eq. (1) for uncoupled states. The red histogram (open bars) in Figs. 2(a) and 2(b) demonstrates the situation for coupled states. Most prominent is the enhanced probability at $\Delta \tilde{s}_{\text {tot }}=0$. Since the red dots in Fig. 22(c) do not agree with the dashed line of slope 1 this apparent entropy production does not obey the FT. Rather a linear relation as given by Eq.(1) with $\alpha \simeq 0.65$ is found.

In additional experiments, we observe linear relations according to Eq. (1) with different slopes $\alpha$, which depend on two parameters: (i) the plasma parameter $\Gamma$, and (ii) the trajectory length $t$, as shown in Figs. 3(a) and 3(b). Clearly, the FT is confirmed for arbitrary trajectory lengths in uncoupled states (black squares in Fig. 3(b)). The obvious dependence of $\alpha$ on $\Gamma$ resembles the transition from an uncoupled to a coupled state. For $\Gamma=300, \alpha$ decays with increasing length $t$, from 1 to 0.65. A similar time dependence has been also observed in [14]; however, there it was not identified as an inherent feature of hidden degrees of freedom. In additional measurements performed for coupling two different NESS, we also found such a linear relation. Therefore, we exclude symmetry as the sole origin of this behavior.

Discussion.-First, we explain why for $t \rightarrow 0$ the slope $\alpha$ approaches 1 . In general, deviations from the FT must be caused by the interaction with the hidden particle. In a short time expansion to lowest order in $t$, we can neglect changes in the interaction force during the motion of the observed particle. Thus, the interaction force entering Eq. (6) through $\tilde{\nu}^{\text {s }}$ becomes constant in this limit. The apparent entropy production then becomes equiv- 
alent to that of an effective one-particle system subject to a Markovian dynamics with mean local velocity $\tilde{\nu}^{\mathrm{s}}$, which trivially obeys the FT. This effective description is valid only to lowest order in time since taking into account higher order terms would include contributions arising from the correlated motion of the observed and the hidden particle.

Although we are able to qualitatively understand the influence of coupling it remains a surprising feature why, in all of our experiments presented so far, only the slope of the FT is affected by the coupling strength while the linear relation Eq. (1) remains untouched. In order to elucidate this result we define the function

$$
f\left(\Delta \tilde{s}_{\text {tot }}\right) \equiv \ln \left[p\left(\Delta \tilde{s}_{\text {tot }}\right) / p\left(-\Delta \tilde{s}_{\text {tot }}\right)\right],
$$

which we assume to be analytic. First, we note that $f$ is antisymmetric by construction, and thus for small entropy productions, $\Delta \tilde{s}_{\text {tot }} \ll 1, f$ trivially must be linear up to corrections of third order or higher [27]. Second, we discuss $f$ for large entropy productions, $\Delta \tilde{s}_{\text {tot }} \gg 1$. Solving Eq. (7) for $p\left(-\Delta \tilde{s}_{\text {tot }}\right)$ and integrating over all $\Delta \tilde{s}_{\text {tot }}$ yields

$$
\int_{-\infty}^{+\infty} p\left(\Delta \tilde{s}_{\text {tot }}\right) e^{-f\left(\Delta \tilde{s}_{\text {tot }}\right)} d \Delta \tilde{s}_{\text {tot }}=1
$$

by normalization. We assume that $p\left(\Delta \tilde{s}_{\text {tot }}\right)$ does not decay faster than a Gaussian as we have observed in all our measurements. For any quantity consisting of independent contributions the central limit theorem would imply a Gaussian. Any correlation will typically lead to an even slower decay. Under this assumption convergence of the integral in Eq. (8) requires that $f\left(\Delta \tilde{s}_{\text {tot }}\right)=\mathcal{O}\left(\Delta \tilde{s}_{\text {tot }}^{2}\right)$. Since, in addition, $f$ is antisymmetric we expect the generic asymptotic behavior to be linear, $f\left(\Delta \tilde{s}_{\text {tot }}\right) \sim$ $\Delta \tilde{s}_{\text {tot }}$, with a slope generally different from the one for small $\Delta \tilde{s}_{\text {tot }}$.

Summarizing these arguments, we expect a linear function both for small and for large entropy production for any time $t$. For intermediate entropy production this reasoning leaves the possibility of a nonlinear behavior. Even though we have found a constant slope for most experimental parameters, by fine-tuning the system and plasma parameter, we can observe an obviously nonlinear result, as shown in Fig. 4(a).

In contrast to the previous data, here, the two particles are subjected to quite different potentials whereas the driving forces remain untouched. The potential of the hidden particle is adjusted such that it circulates freely along the torus $\left(V_{2}^{0}=71 \mathrm{k}_{\mathrm{B}} \mathrm{T}\right)$ whereas a deep minimum $\left(V_{1}^{0}=262 \mathrm{k}_{\mathrm{B}} \mathrm{T}\right)$ remains in the tilted potential $U_{1}$ of the observed particle, which, for $\Gamma=0$, it is not able to leave. The latter's motion sets in only when the coupling helps it to surmount the potential barrier. This mode is identified in a typical trajectory shown in Fig. 4(b). The hidden particle (gray/thin line) moves with a period of
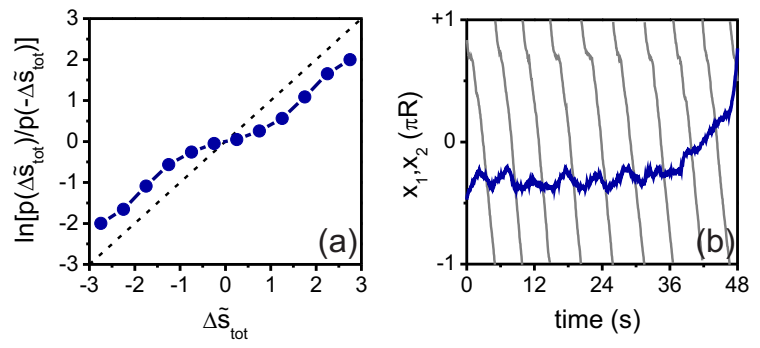

FIG. 4: (color online) (a) $\ln \left[p\left(\Delta \tilde{s}_{\text {tot }}\right) / p\left(-\Delta \tilde{s}_{\text {tot }}\right)\right]$ as a function of $\Delta \tilde{s}_{\text {tot }}$ for $t=3 \mathrm{~s}$. (b) Typical trajectories of the observed (blue/thick line) and hidden particle (gray/thin line).

$4 \mathrm{~s}$ and almost constant velocity along $U_{2}\left(x_{2}\right)$. Around $x_{2}=0.75$ it slightly slows down due to interaction. The reaction of the observed particle (blue/thick line) is more pronounced since locally it is confined within a potential minimum at $x_{1}=-0.35$ and there the interaction forces are dominant displacing it along positive $x_{1}$. The apparent oscillations in the trajectory originate from the fact that not every time the observed particle is pushed (by the hidden one) this action results in a surmounting of the potential barrier. In most of the cases, the particle just relaxes to its original position. We observe that nonlinearities in the intermediate regime of $\Delta \tilde{s}_{\text {tot }}$ are most pronounced when the trajectory length matches approximately the oscillation period.

Concluding Perspectives.-We have investigated the influence of hidden slow degrees of freedom on the FT. In our experiments, we typically find that a FT-like symmetry is preserved, however, with a different slope which depends, in particular, on the length of the observed trajectories. Consequently, in any experiment, where hidden slow degrees of freedom cannot be ruled out a priori, an observed linear behavior cannot be used to infer quantities by implicitly assuming $\alpha=1$. Theoretically, we have argued that a slope of 1 is to be expected for short trajectories while, for any length, for both small and large entropy production a constant slope should be generic. Classifying theoretically the conditions for finding an almost constant slope over the full range, as we often did in our experiments, remains a task for future work. Likewise, it will be important to explore, both in theory and experiments, how hidden slow degrees of freedom affect other quantities, like work and heat, their exact relations, and the fluctuation-dissipation theorem for NESS.

We thank C. Groben for characterizing the particles and D. Hartich and J. R. Gomez-Solano for interesting discussions. V. B. was supported by the Deutsche Forschungsgemeinschaft (Grant No. BL 1067). 
[1] D. J. Evans, E. G. D. Cohen, and G. P. Morriss, Phys. Rev. Lett. 71, 2401 (1993).

[2] G. Gallavotti and E. G. D. Cohen, Phys. Rev. Lett. 74, 2694 (1995).

[3] J. Kurchan, J. Physics A 31, 3719 (1998).

[4] J. L. Lebowitz and H. Spohn, J. Stat. Phys. 95, 333 (1999).

[5] U. Seifert, Phys. Rev. Lett. 95, 040602 (2005).

[6] G. M. Wang, E. M. Sevick, E. Mittag, D. J. Searles, and D. J. Evans, Phys. Rev. Lett. 89, 050601 (2002).

[7] T. Speck, V. Blickle, C. Bechinger, and U. Seifert, Europhys. Lett. 79, 30002 (2007).

[8] F. Douarche, S. Joubaud, N. B. Garnier, A. Petrosyan, and S. Ciliberto, Phys. Rev. Lett. 97, 140603 (2006).

[9] S. Ciliberto and C. Laroche, J. Phys. IV (France) 08, Pr6-215 (1998).

[10] S. Ciliberto, N. Garnier, S. Hernandez, C. Lacpatia, J.F. Pinton, and G. R. Chavarria, Physica (Amsterdam) 340A, 240 (2004).

[11] K. Feitosa and N. Menon, Phys. Rev. Lett. 92, 164301 (2004).

[12] N. Kumar, S. Ramaswamy, and A. K. Sood, Phys. Rev. Lett. 106, 118001 (2011).

[13] S. Aumaître, S. Fauve, S. McNamara, and P. Poggi, Eur. Phys. J. B 19, 449 (2001).

[14] K. Hayashi, H. Ueno, R. Iino, and H. Noji, Phys. Rev.
Lett. 104, 218103 (2010).

[15] R. Suzuki, H.-R. Jiang, and M. Sano, arXiv:1104.5607.

[16] S. Rahav and C. Jarzynski, J. Stat. Mech. (2007) P09012.

[17] A. Puglisi, S. Pigolotti, L. Rondoni, and A. Vulpiani, J. Stat. Mech. (2010) P05015.

[18] M. Esposito, Phys. Rev. E 85, 041125 (2012).

[19] R. Kawai, J. M. R. Parrondo, and C. Van den Broeck, Phys. Rev. Lett. 98, 080602 (2007).

[20] Y. Utsumi, D. S. Golubev, M. Marthaler, K. Saito, T. Fujisawa, and G. Schön, Phys. Rev. B 81, 125331 (2010).

[21] G. B. Bulnes Cuetara, M. Esposito, and P. Gaspard, Phys. Rev. B 84, 165114 (2011).

[22] S. Ganeshan and N. A. Sinitsyn, Phys. Rev. B 84, 245405 (2011).

[23] L. P. Faucheux, G. Stolovitzky, and A. Libchaber, Phys. Rev. E 51, 5239 (1995).

[24] J. Mehl, V. Blickle, U. Seifert, and C. Bechinger, Phys. Rev. E 82, 032401 (2010).

[25] C. Lutz, M. Reichert, H. Stark, and C. Bechinger, Europhys. Lett. 74, 719 (2006).

[26] V. Blickle, T. Speck, U. Seifert, and C. Bechinger, Phys. Rev. E 75, 060101(R) (2007).

[27] A. Puglisi, P. Visco, A. Barrat, E. Trizac, and F. van Wijland, Phys. Rev. Lett. 95, 110202 (2005).

[28] We determine $\gamma$ and $m_{0}$ by measuring the drag force when a particle is subjected to a magnetic field gradient. 